\title{
Processus de relativisation en bàsàa: de la syntaxe à la prosodie $^{1}$
}

\author{
Emmanuel-Moselly Makasso \\ ZAS
}

Cet article propose une réflexion sur la manière dont la langue bàsàa (Bantu $\mathrm{A} 43$ parlée au Cameroun) exprime la relativisation. En l'absence d'une classe grammaticale de pronoms relatifs la langue utilise la classe des démonstratifs. La stratégie démonstrative mise en place peut selon les cas, associer la classe des locatifs pour déterminer les degrés de définitude. La langue distingue également les relatives restrictives des relatives non-restrictives qui sont soit descriptives, soit emphatiques. Du point de vue prosodique, la fin de la relative en bàsàa coïncide avec une finale de Groupe Intonatif.

This article provides a sketch of the morphosyntax and prosody of relative clauses in Bàsàa, a Bantu language (A 43) spoken in Cameroon. The language does not have a class of relative pronouns like French or English. Rather, the language borrows from the demonstrative class to form relatives. Also, the language can involve locatives in the demonstrative strategy to express relative definiteness. Two varieties of relatives can be found in Bàsàa: restrictive relatives and nonrestrictive relatives, which can be descriptive or emphatic. Prosodically, we find an Intonational Phrase boundary at the end of the relative clause.

\section{Introduction}

Le Bàsàa est une langue Bantu (A 43) parlée au Cameroun, en Afrique Centrale. Le nombre de locuteurs de cette langue est estimé à environ 230000 personnes installées dans les régions du Centre, du Littoral et du Sud.

1 Cette recherche a été menée dans le cadre du projet ANR/DFG BANTUPSYN. Je tiens à exprimer mon infinie reconnaissance à Laura Downing qui m'a fourni un questionnaire sur les relatives, et au SynPhonI working group (Annie Rialland, Elisabeth Selkirk, Cédric Patin, Martial Embanga, Fatima Hamlaoui, Chang Yang Shi) pour leurs suggestions ainsi qu'à Madeleine Ngo Ndjeyiha pour la documentation qu'elle a bien voulu mettre à ma disposition. 
Comparativement à la vaste majorité des langues camerounaises, le bàsàa est assez bien décrit (Bot ba Njock 1970, Dimmendaal 1988, Hyman 2003, Ngo Ndjeyiha 2006, Makasso 2008, entre autres).

Dans cette réflexion, nous traitons de la relativisation en bàsàa, qui, pour s'exprimer, convoque des éléments aussi bien morphosyntaxiques qu'intonatifs. La relativisation dans cette langue a été analysée par Madeleine Ngo Ndjeyiha dans sa thèse de doctorat (2006). Cette auteure reconnaît en bàsàa 6 possibilités pour exprimer les relatives:

- Présentatif + Nom + Démonstratif $+\ldots$

- Présentatif + Nom $+\ldots$

- Présentatif + Nom + Démonstratif $+\mathrm{Pa}+$ locution relative $+\ldots$

- Démonstratif + Nom $+\ldots$

- $\mathrm{Nom}+\mathrm{Nom}+\ldots$

- Nom + Complémenteur.

Notre point de vue est que la langue bàsàa dispose, en fait, d'une seule stratégie morphosyntaxique lui permettant d'exprimer la relativisation: la stratégie démonstrative. Comme le bàsàa ne possède pas de classe de pronoms relatifs comme en français ou en anglais, le processus de relativisation utilise des marques qui ne lui sont pas propres, mais sont empruntées à la classe des démonstratifs. Cet article vise donc principalement à montrer comment les démonstratifs s'emploient pour construire des relatives, lesquelles impliquent aussi certains paramètres prosodiques.

Cet article se structure de la manière suivante: après une section 2 consacrée à la description des caractéristiques morphosyntaxiques de la relativisation, la section 3 initie une réflexion sur le sens à donner au démonstratif dans ce processus. La section 4 s'intéresse à la prosodie dans la construction des relatives.

\section{Caractéristiques morphosyntaxiques de la relativisation en bàsàa}

\subsection{Le démonstratif en bàsàa}

Trois références spatiales peuvent être relevées en bàsàa concernant la monstration :
(a) Près du locuteur,
(b) Près du récepteur,
(c) Loin du locuteur et du récepteur.

La deuxième catégorie est celle qui sert dans le processus de relativisation. Le démonstratif peut précéder ou suivre le nominal qu'il modifie et, dans un cas comme dans l'autre, il s'accorde selon la classe du nom. Il est alors adnominal. 
Mais il peut être pronominal quand il se réalise isolément, sans le nom. Il importe cependant de noter que les démonstratifs adnominal et pronominal ont les mêmes formes en bàsàa.

\section{Premier cas:}

\section{Dem + Nom}

Quand le Nom est précédé du démonstratif, il s'agit d'un démonstratif adnominal. La forme pronominale qui rend l'idée de celui-ci ou celui-là n'est pas de la forme Dem+nom. Ainsi, l'exemple (1) suivant, comporte une forme adnominale /adjectivale du démonstratif avec cet enfant-ci.

(1) núnú màngé à ńdzòp

Dem enfant acc pr-entrer

'Cet enfant-ci rentre.'

(2) núnú à ńdzòp núnú à ńdzòp béé

Dem acc pr-entrer Dem acc pr-entrer neg

'Celui-ci rentre, celui-ci ne rentre pas.'

$\begin{array}{lllll}\text { mè } & \text { y’!gwés } & \text { ìní !ndáp } & \text { lél } & \text { ìí } \\ \text { je } & \text { pr-aimer } & \text { Demmaison } & \text { dépasser } & \text { Dem }\end{array}$

'Je préfère cette maison-ci à celle-là.'

L'énoncé (2) présente deux fois le démonstratif pronominal núnú celui-ci qui se substitue au groupe nominal núnú màngé cet enfant-ci. L'énoncé (3) présente également un démonstratif pronominal ìi celle-là.

Tableau 1: distribution spatiale du démonstratif préposé

\begin{tabular}{|c|c|c|}
\hline Près du locuteur & Près du récepteur & Loin des deux \\
\hline $\begin{array}{c}\text { núnú mùràa } \\
\text { cette femme-ci }\end{array}$ & $\begin{array}{c}\text { nú mùràá } \\
\text { cette femme-là }\end{array}$ & $\begin{array}{c}\text { núú mùráá } \\
\text { cette femme là-bas }\end{array}$ \\
\hline $\begin{array}{c}\text { ìní !ndáp } \\
\text { cette maison-ci }\end{array}$ & $\begin{array}{c}\text { ì ndáp } \\
\text { cette maison-là }\end{array}$ & $\begin{array}{c}\text { ì́ !ndáp } \\
\text { cette maison là-bas }\end{array}$ \\
\hline híní hìlóyá & hí hìlóyá & híí hìlóyá \\
ce garçon-ci & ce garcon-là & ce garcon là-bas \\
\hline
\end{tabular}

Deuxième cas:

$$
\text { í + Nom + Dem }
$$


Le nom s'insère dans une construction de type 1 .........Dem. Cette construction qui est de nature adnominale montre comment le nom s'associe à la marque du locatif $i$ et au pronom démonstratif.

Tableau 2: distribution spatiale du démonstratif postposé

\begin{tabular}{|c|c|c|}
\hline Près du locuteur & Près du récepteur & Loin des deux \\
\hline $\begin{array}{l}\text { í mùràá núnú } \\
\text { cette femme-ci }\end{array}$ & $\begin{array}{c}\text { í mùràá nû } \\
\text { cette femme-là }\end{array}$ & $\begin{array}{c}\text { í mùràá núú } \\
\text { cette femme là-bas }\end{array}$ \\
\hline $\begin{array}{c}\text { í !ndáp ìni } \\
\text { cette maison-ci }\end{array}$ & $\begin{array}{c}\text { í !ndáp ì } \\
\text { cette maison-là }\end{array}$ & $\begin{array}{c}\text { í !ndáp ìí } \\
\text { cette maison là-bas }\end{array}$ \\
\hline $\begin{array}{c}\text { híłlóyá (î hìlóyá) híní } \\
\text { ce garçon-ci }\end{array}$ & $\begin{array}{l}\text { híłlóyá (î hìlóyá) hî } \\
\text { ce garcon-là }\end{array}$ & $\begin{array}{c}\text { híl1’oyá (í hìlóyá) híi } \\
\text { ce garcon là-bas }\end{array}$ \\
\hline
\end{tabular}

La nature de la particule $\hat{i}^{2}$ suscite beaucoup de controverses. C'est d'abord Dimmendaal (1988: 58), suivi par Hyman (2003) qui l'ont analysée. Pour ces auteurs, le préfixe nominal de classe acquiert un ton haut quand le démonstratif est postposé. Ainsi, devant un nom à préfixe 0 , un $\mathfrak{F}$ - sous-jacent apparaît. Cette position n'éclaire ni sur la nature, ni sur la fonction de la particule.

Par ailleurs, Van de Velde (2005) pose que le démonstratif en bàsàa est essentiellement adnominal, étant donné qu'il existerait un augment rattaché au nom. Cela voudrait dire, selon lui, que pour le cas de hílóyá híní "ce garçonci", le démonstratif adnominal híní étant postposé, le ton flottant haut de l'augment se rattacherait au nom qui ainsi, présenterait un comportement différent de la version híní hil óyá “ce garçon-ci”. Le problème de cette position est que l'augment, s'il a existé en bàsàa, a totalement disparu. La morphologie nominale du bàsàa comporte des préfixes nominaux, mais pas de pré-préfixe ou augment. Le démonstratif dans notre analyse est soit adnominal quand il est modificateur d'un nom, soit pronominal quand il est utilisé isolément.

Sur un plan purement dialectal, la partie sud du territoire bàsàa, le pays des Babimbi qui, d'après la légende, sont restés près du rocher dont les Bàsàa sont issus, est considéré comme très conservatrice d'un point de vue linguistique. La forme híl'óyá hî' "ce garçon-ci" n'est pas facilement acceptée chez eux, on trouve plutôt í hilóyá hî pour "ce garçon-ci". Cet usage montre bel et bien l'existence de cette particule dans la construction du démonstratif. Ngo Ndjeyiha (2006) a dénommé cette particule le présentatif, mais de notre point de vue, et nous le verrons ci-dessus, il s'agit d'une marque du locatif.

2 La particule du locatif porte un ton bas flottant après le ton haut et se transcrit phonologiquement / í-'/. C'est à cause de ce ton bas flottant qu'il y a des downsteps sur les premières syllables à ton haut des mots adjacents. 


\subsection{Le locatif dans la construction démonstrative en bàsàa?}

Comme beaucoup de langues bantoues, la langue bàsàa possède une classe de locatifs. Les locatifs expriment la localisation dans l'espace ou le temps, le lieu ou le moment où se déroule le procès véhiculé par le verbe.

$\begin{array}{lllll}1 & \text { bòm au marché } & 1 & \text { ndzèl } & \text { en route } \\ 1 & \text { ygì } a u-d e s s u s & 1 & \text { kòsí } & \text { à midi }\end{array}$

Phonologiquement, le marqueur du locatif / í -/ a un ton bas flottant après son ton haut. Ainsi, quand le nominal qui le suit porte un ton haut sur la première syllabe, il se produit un downstep.
í ‘ndáp
à la maison
‘pán
près

S'agissant de la construction démonstrative, le locatif peut subir ou occasionner des transformations dans son environnement phonologique. Dans le cas de híl'́yá hî "ce garçon", deux processus phonologiques ont été réalisés : d'abord, la particule /í-/ a fusionné avec le préfixe nominal [hì-], amenant un ton haut sur ce-dernier. Ensuite, le ton bas flottant du locatif fusionné avec le préfixe nominal cause un downstep sur la première syllabe du radical nominal.

\subsection{Usages anaphorique et déictique du démonstratif en bàsàa?}

La fonction principale du démonstratif est d'attirer l'attention du récepteur sur des éléments autour de lui. Il peut aussi s'agir d'éléments déjà présents dans le contexte linguistique ou dans sa mémoire. D'après une distinction proposée par Himmelmann (1996) et Diessel (2006), il existe deux interprétations pragmatiques possibles du démonstratif: l'usage anaphorique et l'usage exophorique. La version Démonstratif + Nom correspond à un usage anaphorique parce que généralement, elle renvoie à une réalité déjà présente dans le contexte linguistique. Ainsi, le groupe nominal [núnú màngé] cet enfantci ne peut se comprendre que s'il a été question des enfants au préalable. Il peut s'agir également de l'expression d'un contraste du genre celui-ci et aucun autre.

L'autre version par contre $(/ \hat{i}-/+$ Nom + Démonstratif) a un usage exophorique ou déictique, car le locuteur indique un élément présent dans le contexte immédiat ou social du récepteur. Cette distinction nous sera utile plus loin lors de l'analyse des relatives. 


\subsection{La stratégie démonstrative}

Les démonstratifs en bàsàa contribuent à restreindre la référence d'un nom, en le localisant dans le contexte spatial ou référentiel lié au locuteur ou au récepteur, ou à tous les deux. Ainsi donc, la langue utilise de manière non-équivoque la stratégie démonstrative pour exprimer les relatives. Plusieurs autres langues bantu se comportent comme le bàsàa (Zeller 2004 pour le Sotho, Demuth et Harford 1999 pour le Sotho, Chumbow 1977 pour le Ngemba, Mutaka 2000 pour le Bafut, Cheng et Kula 2006 pour le Bemba).

Dans les sections suivantes, nous verrons comment la relative en bàsàa s'exprime à travers le démonstratif. Il faut toutefois noter que la relative ne comporte pas toujours le démonstratif post-nominal qui est facultatif. Le démonstratif dans les énoncés ci-dessous sera marqué Dem, et le locatif sera marqué LoC.

\subsubsection{Relatives sujets ou objets}

Qu'il s'agisse des relatives en fonction sujet ou objet, la marque formelle est la même. La seule différence repose sur les accords entre l'antécédent et la proposition principale. Pour les relatives sujets (cf. (6) et (7)), l'accord est le même entre la relative et la principale car le sujet est le même, ce qui n'est pas le cas pour les relatives objets (cf. (8) et (9)).
(6) í bòră
(bá)
6á ǹ-t纴 $h \hat{\varepsilon} \quad w \hat{\varepsilon}$
bá ́-kè
í !nhóyá
Loc femmes Dem acc p1-voir 2sg-obj
acc pr-partir loc nager
'Les femmes qui t'ont vu vont nager.'
í gwǒm (ßí)
mè
m̀̀-6éyèl
Bí ńn-jèr
Loc choses Dem
1sg-subj p1-porter
3pl-subj pr-peser
'Les choses que j'ai portées pèsent lourd.'

La présence du Dem (entre parenthèses) est facultative. De même, on voit pour les relatives sujets, que le même morphème d'accord sert de sujet pour la principale et pour la subordonnée.
(8)
í Gòră (bá)

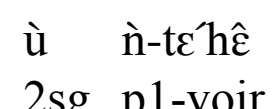
6á $\quad y^{\prime}-k \grave{c}$
í g’!hóyá
Loc femmes Dem 2sg p1-voir 3pl pr-partir loc nager
'Les femmes que tu as vues vont nager.' 


\begin{tabular}{|c|c|c|c|c|}
\hline $\begin{array}{l}\text { mè } \quad y^{\prime}-{ }^{+} \text {gwés } \\
\text { 1sg-subj pr-aimer }\end{array}$ & $\begin{array}{l}\text { í } \quad \text { bòm } \\
\text { Loc marché }\end{array}$ & $\begin{array}{l}\text { (í) } \\
\text { Dem }\end{array}$ & $\begin{array}{l}\text { 6á } \\
3 \text { pl-subj }\end{array}$ & $\begin{array}{ll}\text { ń-tìp } & \text { on } \\
\text { nr- finir } & \text { bâtir }\end{array}$ \\
\hline
\end{tabular}

On voit bien que la présence du Dem (entre parenthèses) est facultative. La différence ici avec les relatives -sujets vient de ce que le sujet n'est pas le même entre la relative et la subordonnée.

\subsubsection{Catégories sémantiques des relatives}

En bàsàa, il existe aussi bien des relatives restrictives que des relatives nonrestrictives. Les relatives restrictives définissent l'antécédent en donnant une information assez détaillée tout en limitant la portée sémantique du mot (antécédent). Les relatives non-restrictives quant à elles apportent des informations additionnelles sur l'antécédent, sans le définir. La différence entre les deux types de relatives est marquée par l'insertion d'une particule emphatique dans la non-restrictive. Les relatives des exemples 10, 11 et 12 ne présentent pas de particule emphatique et elles donnent lieu à une interprétation restrictive :

(10) bòngé (6á) ‘bá ń-`’jí hók bá ń-là tûk í páy !lép Enfants Dem acc pr-savoir nager acc pr-pouvoir jouer loc bord rivière 'Les enfants qui savent nager peuvent jouer près de la rivière.'
(11) ndż̀̀
ì ń-sèndì ì
ý-àp
ygándàk
Chemin Dem acc pr-glisser acc pr-allonger beaucoup
'Une route qui glisse parait de plus en plus longue.'

Pour que les enfants puissent jouer au bord de la rivière, ils doivent avoir une caractéristique qui les définit: savoir nager. De même, la route n'est pas simplement longue, elle glisse aussi.

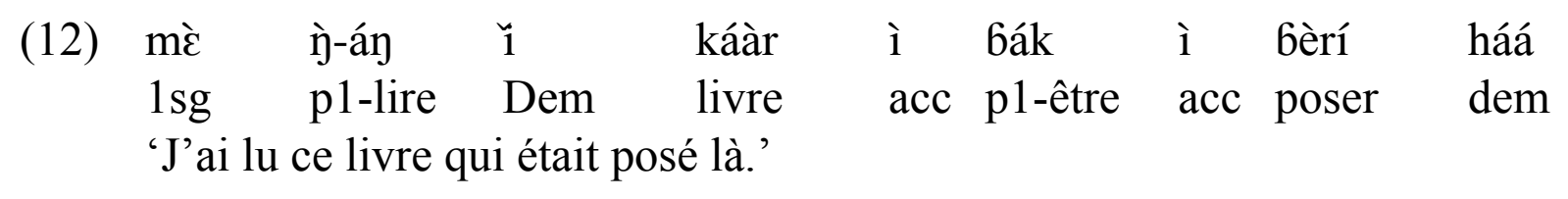

Evidemment, le livre qui était posé là est différent des autres livres tout autour de par sa position. Et c'est celui-là précisément qui a été lu. Ainsi, ce livre est défini par rapport à sa position. Les énoncés 13 et 14 suivants ont une interprétation non-restrictive, du fait de la présence en leur sein d'une particule emphatique : 


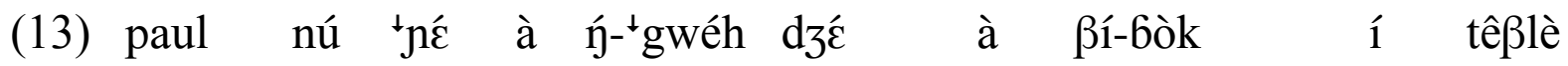
Paul Dememph acc pr-aimer manger acc p2-être 1er loc table 'Paul, qui aime manger, était le premier à table.'

(14) Gàúrú 6êm bá ‘bó bá ßí-`sál lóngê bá ßí-nc̀r bóbasô élèves poss Dem emphaccp2-travailler bien acc p2-réussir tous 'Mes élèves, qui ont bien travaillé, ont tous réussi.'

Les énoncés en (13) présentent une emphase sur le nom (antécédent de la relative). Les particules emphatiques utilisées: [nع'] pour (13) et [6’o] pour (14) attirent l'attention sur le nom et plutôt que de le définir, le décrivent. Ainsi, dans (13), il ne s'agit pas d'un Paul parmi d'autres qui aimerait manger, il s'agit d'un Paul spécifique.

\subsubsection{Autres relatives}

Comme c'est le cas dans beaucoup d'autres langues Bantu, le bàsàa peut relativiser des noms dont la référence est possessive ou locative. Il y a alors introduction d'un marqueur résomptif.

(15) í mùr ygònd jèé ì ý-kòn à ý-kèná jó í dôktà Loc homme fille poss acc pr-malade acc pr-amener 3sg loc hôpital 'L'homme dont la fille est malade est entrain de la conduire à l'hôpital.'

Dans l'énoncé ci-dessus, nous avons une relative possessive. Le résomptif présenté ici est jèé.

(16) paul à m̀-bíi káàr í ggìí têßlè Paul acc p1-poser livre loc dessus table 'Paul a pose le livre sur la table.'

(17) í têßlè paul à m̀-bíi káàr í ygùì (jéłé) í m’-pùm Loc table Paul acc p1-poser livre loc dessus poss acc pr-être sale 'La table sur laquelle Paul a pose le livre est sale.'

L'énoncé (17) présente une relative locative qui combine aussi bien le locatif résomptif (loc+dessus) que le possessif résomptif. Il est important de noter ici que dans cette phrase, le possessif résomptif n'est pas obligatoire. 


\section{Sémantique des relatives}

Notre objectif dans cette section est de tenter d'expliquer comment le locuteur bàsàa conçoit la relativisation. Dans le cadre de la restriction de la référence d'un nom comme le propose la définition de la relative, le locuteur bàsàa fixe cette référence sur un espace, convoquant les sens ou l'attention de son allocutaire (ce que nous avons appelé plus haut l'usage anaphorique ou exophorique de la relative). Les relatives en bàsàa peuvent se classer selon leur degré de définitude.

\subsection{Quand l'antécédent est défini}

Nous avons une structure comme suit:

$$
\text { Loc }+ \text { Nom }+(\text { Dem })+\text { acc }+ \text { relative }
$$

La notion de définitude telle qu'utilisée ici renvoie à la présence (actuelle ou virtuelle) de la référence du nom, ou de son absence complète. Quand il y a la particule locative [í] qui accompagne le démonstratif, l'attention du récepteur est attirée sur une réalité qui existe quelque part. Ceci se rapporte à la notion de démonstratif à usage exophorique, qui attire l'attention de l'auditeur sur un élément présent dans le contexte immédiat ou social.

(18) í 6òngé 6á ‘bá ń- ’’jí hók bá ń-tùk í ‘pán lép Loc enfants Rel acc pr-savoir nager acc pr-jouer loc côté rivière 'Les enfants qui savent nager jouent près de la rivière.'

$\begin{array}{llllllll}\text { (19) } & \text { mè } & \text { ỳ-án } & 1 & \text { kààr } & \breve{1} \quad \text { ì bèrí } & \text { háá } \\ \text { 1sg } & \text { p1-lire } & \text { Loc livre } & \text { Dem acc } & \text { statif-poser } & \text { Dem } \\ \text { 'J'ai lu le livre qui est posé là.' } & & & & \end{array}$

Dans (18), on considère un groupe d'enfants précis, que la caractéristique de "savoir nager" distingue d'autres enfants. De même dans l'énoncé (19), on doit voir le livre que le locuteur indique avec son doigt.

\subsection{Quand l'antécédent n'est pas défini}

La non-définitude peut être entendue comme une absence de restriction, une généralité. En somme, il s'agit d'une réalité englobant le contexte immédiat et le 
rattachant à un univers plus vaste. La structure de la relative non-définie est la suivante:

$$
\text { Nom + ( Dem) + acc + Relative }
$$

bòngé bá 'Đá ń-’’jí hók bá ń-là tûk í !páy lép
Enfants Dem acc pr-savoir nager acc pr-pouvoir jouer loc côté rivière 'Les enfants qui savent nager peuvent jouer au bord de la rivière.'

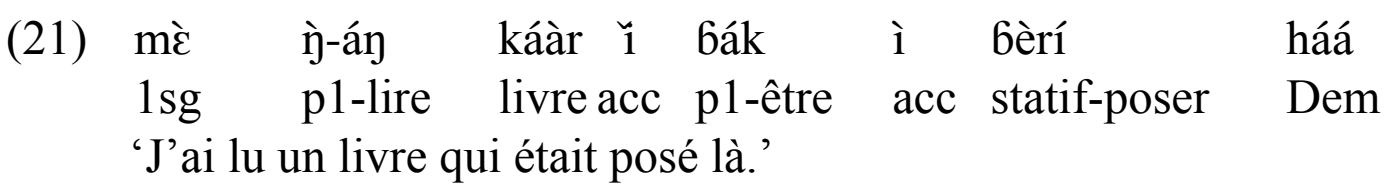

Dans (20), il s'agit de n'importe quel enfant. C'est sans limite déterminée, car non-défini. Tout enfant sachant nager peut jouer près de la rivière. On ne voit ni n'imagine pas un groupe d'enfants précis. De même, (21) ne renvoie pas à un livre précis, et probablement que le récepteur n'est pas au courant de l'existence même de ce livre.

On voit bien que le démonstratif post-nominal est facultatif, qu'il s'agisse d'un antécédent défini ou pas. Toutefois, dans une relative enchâssée, le démonstratif post-nominal est obligatoire pour séparer les deux relatives, comme nous pouvons le voir dans (22) ci-dessous.

(22) í mùră (nú) mè pórlák nú à ßí-!sómb wóm...

Loc femme Rel 1sg ppr-parler dem acc p1-acheter champ

'La femme dont je te parlais, qui a acheté un champ...'

凅 mùră mè pórlák à $\beta$ í-!sómb wóm...

Rel femme 1sg ppr-parler acc p1-acheter champ

'La femme dont je te parlais, a acheté un champ...'

On voit à l'exemple ci-dessus que l'absence du démonstratif amène à envisager une relative simple.

\section{Prosodie des relatives}

Une caractéristique importante des relatives en bàsàa est leur organisation prosodique, en particulier le fait que leur frontière droite coïncide avec la fin d'un Groupe Intonatif. Le corpus utilisé pour la prosodie des relatives est constitué d'un enregistrement de parole lue de deux locuteurs (une femme et un homme) du questionnaire sur les relatives développé autour du projet BANTUPSYN. 
La finale de Groupe Intonatif est, entre autres marquées par des réalisations tonales caractéristiques. Considérons le cas du pronom $W \varepsilon^{2}$ "toi" et de ses diverses réalisations en fonction des contextes. Au sein du Groupe Intonatif et en dehors de la position objet suivant immédiatement le verbe, il porte simplement son ton bas lexical, comme l'illustre l'exemple (23).
mè bák ní wè í bòm
$1 \mathrm{sg}$ être avec toi loc marché
'J'étais avec toi au marché.'

Il reçoit un ton haut lorsqu'il est objet immédiatement après le verbe, son ton bas lexical devenant flottant. Ce dernier provoque le downstep lorsqu'un ton haut suit comme dans l'exemple (24)

$\begin{array}{llll}\text { mè } & \text { pórlák } & \text { wé } & \text { !kóp } \\ \text { 1sg } & \text { ppr-parler } & \text { 2sg obj } & \text { poulet } \\ \text { 'Je te parlais d'un poulet.' } & \end{array}$

Devant un mot à ton bas, ce même ton bas n'est pas réalisé, comme dans l'exemple suivant.
(25) mè pórlák wé mùră 1 sg ppr-parler 2sg obj femme 'Je te parlais d'une femme.'

En finale d'énoncé de $W \hat{\varepsilon}$ porte un contour descendant, car le ton bas lexical se réalise.
mè pórlák $\quad \mathrm{w} \hat{\varepsilon}$
1sgppr-parler 2sg obj acc p1-acheter champ
'je te parlais'

Dans le cas de la relative en (27), la réalisation de $w \hat{\varepsilon}$ est la même qu'en fin de l'énoncé (26). Cela démontre que la fin de la relative coïncide avec la fin d'un Groupe Intonatif.
(27)

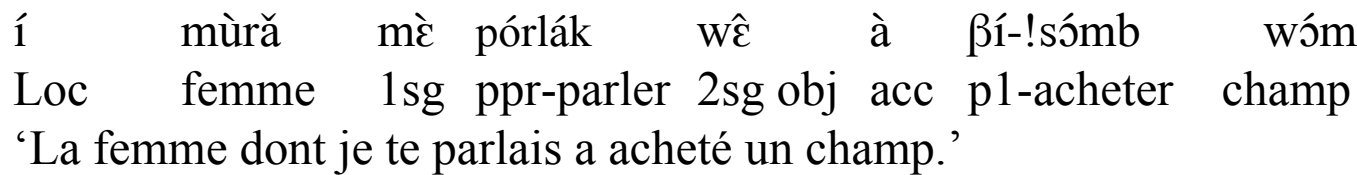


En bàsàa les Groupes Intonatifs sont également séparés par des pauses. Dans notre corpus, les Groupes Intonatifs (entre la proposition principale et la relative) convoquent une pause majeure à $97 \%$ des cas, ainsi que l'illustre la figure ci-après:
1 $\quad$ !kél
rì
ßí-jèyà
ì m̀-pám
Loc jour
nous
p2-entendre
acc p1-sortir

'Le jour qu'on a prévu est arrivé.'

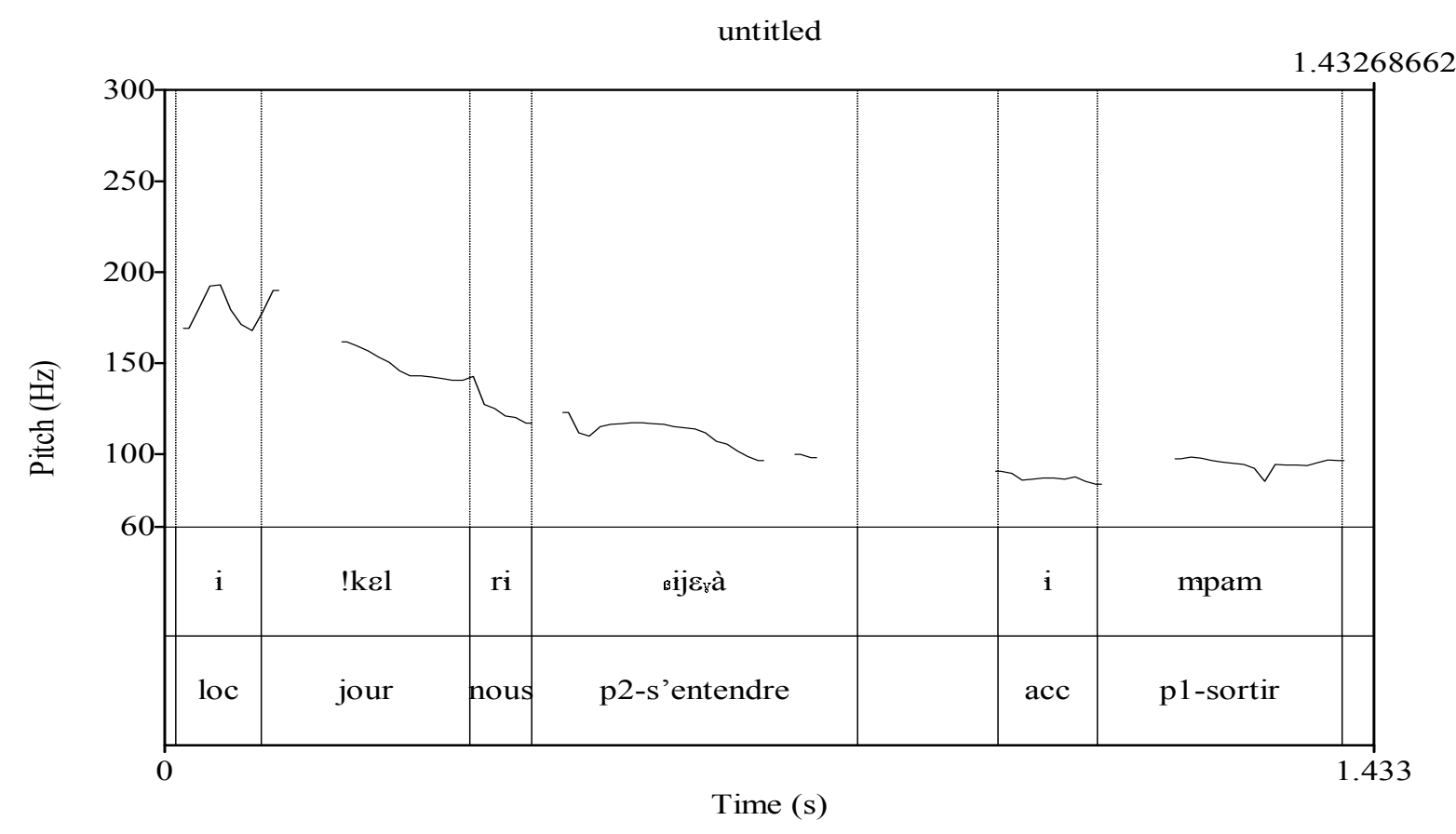

\section{Conclusion}

Dans cette réflexion, il était question de déterminer la manière dont la langue bàsàa exprime la relativisation. La langue ne dispose pas d'une classe de pronoms relatifs, elle adapte une stratégie basée sur l'utilisation du démonstratif. La langue distingue des relatives restrictives des relatives non-restrictives marquées par un pronom emphatique. La langue permet également de déterminer le degré de définitude des relatives marqué notamment par la présence ou l'absence du locatif avant le nominal antécédent. Par ailleurs, du point de vue prosodique, la fin d'une proposition relative coïncide le plus souvent avec une finale de Groupe Intonatif. 


\section{Références}

Bot ba Njock, H. M. (1970). Nexus et nominaux en bàsàa, Thèse de doctorat d'Etat, Université de Paris III - Sorbonne.

Bodomo, A. \& K. Hiraiwa (2004). Relativization in Dagaare, Journal of Dagaare Studies, Vol. 4, 53-75.

Cheng, L. L. (2006). Decomposing Bantu Relatives, NELS 36, Christopher Davis, Amy Rose Deal, and Youri Zabbal (eds.), 197-215.

Cheng, L \& N. C. Kula (2006). Syntactic and phonological phrasing in Bemba Relatives, ZAS Papers in Linguistics vol. 43, pp. 31-54.

Chumbow, S. B. (1977). Relatives as determiners: A case from Ngemba. In Kotey, P. F. \& Der-Houssikian, H. (eds.) Language and Linguistic problems in Africa. Columbia: Hornbeam Press Incorporated, 283-302.

Comrie, B. \& T. Kuteva (2001). Relativization strategies, in Haspelmath, M., Dryer, M. S., David Gil, D. \& Comrie, B. (eds.), The World Atlas of Language Structures. Oxford: Oxford University Press, 494-501.

Creissels, D. (2006). Syntaxe générale une introduction typologique 2 : la phrase. 2/2, Paris, Hermès - Lavoisier.

Demuth, K. \& C. Harford (1999). Verb raising and subject inversion in Bantu relatives. Journal of African Languages and Linguistics. 20, 41-61.

De Vries, M. (2005) The Fall and Rise of Universals on Relativization, Journal of Universal Language 6, 1-33.

Diessel, H. (2006). Demonstratives, joint attention, and the emergence of grammar, Cognitive Linguistics 17 (4), 463-489.

Dimmendaal, G. (1988). Aspects du basaa (Bantou Zone A, Cameroun), Trad. L. Bouquiaux, Paris, SELAF, $92 \mathrm{p}$.

Frota, S., M. D'Imperio, G. Elordieta, P. Prieto \& M. Vigário (2007). The Phonetics and Phonology of Intonational Phrasing in Romance. In Prieto, P., J. Mascaró \& M.-J. Solé (eds.), Segmental and prosodic issues in Romance Phonology, Amsterdam: John Benjamins, 131-154.

Himmelmann, N. (1996). Demonstratives in narrative discourse: A taxonomy of universal uses. In Fox, B. (ed.) Studies in Anaphora. Amsterdam: John Benjamins. 205-254.

Hyman, L. (2003). Bàsàa A 43. In Nurse, D. \& Philippson, G., (eds.), The Bantu Languages, London: Routledge/Curzon, 257-282.

Kula, N. \& L. Marten (2007). eds. SOAS working Papers in Linguistics, Vol 15. SOAS, Department of Linguistics, School of Oriental and African Studies, University of London.

Makasso, E. M. (2008). Intonation et mélismes dans le discours oral spontané en bàsàa, Thèse de doctorat, Université de Provence (Aix-Marseille 1).

Mutaka, N. (2000). An Introduction to African Linguistics. Lincom Europa: München. 
Ngo Ndjeyiha, M. (2006). La théorie de l'optimalité et quelques aspects de la syntaxe du bàsàa (Bantu A 43). Thèse de doctorat, Université de Yaoundé 1.

Van de Velde, M. (2005). The order of noun and demonstrative in Bantu. In K. Bostoen \& J. Maniacky (eds.), Studies in African comparative linguistics with Special Focus on Bantu and Mande. Tervuren, 425-441.

Watters, J. R. (2000). Syntax. In B. Heine \& D. Nurse (eds.), African Languages: An Introduction. Cambridge: Cambridge University Press, 194-230.

Welmers, W. E. (1973). African Language Structures. Berkeley: University of California Press.

Zeller, J. (2004). Relative clause formation in the Bantu languages of South Africa. Southern African linguistics and Applied Language Studies 22 (1\& 2), 75-93. 